\title{
Edge Preserving Image Fusion Based on Contourlet Transform
}

\author{
Ashish Khare, Richa Srivastava, and Rajiv Singh \\ Department of Electronics \& Communication, \\ University of Allahabad, Allahabad, India \\ ashishkhare@hotmail.com, \{gaur.richa,jkrajivsingh\}@gmail.com
}

\begin{abstract}
Image fusion is an emerging area of research having a number of applications in medical imaging, remote sensing, satellite imaging, target tracking, concealed weapon detection and biometrics. In the present work, we have proposed a new edge preserving image fusion method based on contourlet transform. As contourlet transform has high directionality and anisotropy, it gives better image representation than wavelet transforms. Also contourlet transform represents salient features of images such as edges, curves and contours in better way. So it is well suited for image fusion. We have performed experiments on several image data sets and results are shown for two datasets of multifocus images and one dataset of medical images. On the basis of experimental results, it was found that performance of proposed fusion method is better than wavelet transform (Discrete wavelet transform and Stationary wavelet transform) based image fusion methods. We have verified the goodness of the proposed fusion algorithm by well known image fusion measures (entropy, standard deviation, mutual information (MI) and $Q_{A B}^{F}$ ). The fusion evaluation parameters also imply that the proposed edge preserving image fusion method is better than wavelet transform (Discrete wavelet transform and Stationary wavelet transform) based image fusion methods.
\end{abstract}

Keywords: Image fusion, Contourlet transform, Wavelet transform, Edge preserving image fusion, Laplacian and directional filter banks.

\section{Introduction}

Image fusion $[1,2]$ is a technique of computer vision that integrates all relevant and complementary information from different source images into a single composite image without introducing any artifact or noise. The objective of image fusion [3] is to combine information from multiple source images which contains the best relevant information coming from source images and is more suitable for human or machine perception.

Image fusion is an interesting area of research having a number of applications in medical imaging [4], remote sensing [5], satellite imaging [6], target tracking [7], concealed weapon detection [8] and biometrics [9]. Most of the imaging sensors are capable to capture a single type of image that provides only a specific kind of 
information. For example, in remote sensing, we have multispectral image with low spatial resolution and high spectral resolution and panchromatic image with high spatial resolution and low spectral resolution. Similarly in medical imaging, Positron Emission Tomography (PET) image provides functional information whereas the Magnetic Resonance Imaging (MRI) image gives anatomical information. These examples explain that a single imaging sensor is not able to provide all relevant information. Hence fusion of different source images is required in order to retrieve desired information in a single image.

Generally image fusion can be classified into three categories [10]: pixel level fusion, feature level fusion and decision level fusion. In the present work, we propose a pixel image fusion technique, as pixel level image fusion is computationally efficient and original quality of images are not lost during fusion process.

Image fusion techniques vary from spatial domain to transform domain. Limitations of spatial domain fusion techniques [11] lead to transform domain fusion techniques. Wavelet transform based fusion methods $[3,12,13,14]$ are widely found in literatures. Discrete Wavelet Transform (DWT) $[15,16]$ and Stationary Wavelet Transform (SWT) [17] are examples of wavelet transform based image fusion methods. But wavelet transform based fusion methods are limited as they can isolate the discontinuities at edges but failed to capture the smoothness along the contours and curves. Also wavelet transform has limited directionality (e.g. two dimensional DWT provides directional information in three orientations: horizontal, vertical and diagonal), that leads to loss of directional information in resulting fused image.

Due to these limitations of wavelet transform, we have used Contourlet Transform (CT) [18-23] for image fusion which provides more directional information and smoothness about contours. In the present work, we have proposed an edge preserving image fusion method using contourlet transform and compared the proposed method with wavelet transform based fusion techniques (DWT and SWT) using different fusion evaluation parameters.

The rest of the paper is organized as follows: Section 2 briefly describes the contourlet transform. In section 3, the proposed fusion method is elaborated. Results and discussions are given in section 4. Finally, conclusions of the work are given in section 5.

\section{The Contourlet Transform}

The Contourlet Transform [18-23] has a rich set of basis functions and it can represent salient features of images such as edges, curves and contours efficiently. It satisfies the basic requirement for image representation and provides a multiresolution representation of images with increased directionality and anisotropy. The construction of contourlet transform follows double filter banks approach, the laplacian pyramid (LP) and the directional filter banks (DFB). These filter banks jointly known as pyramidal directional filter banks (PDFB).

The contourlet transform is performed in two steps. First is subband decomposition and second is directional transform. The laplacian pyramid is used to capture the point discontinuities and the directional filter bank is used to link the point discontinuities 
into linear structures. Thus the filter banks used in contourlet transform gives a detailed high directional image representation and captures intrinsic geometric structures as well. Hence it is well suited for image fusion with increased directionality and smooth representations along edges, curves and contours. The contourlet transform framework is shown in figure 1.

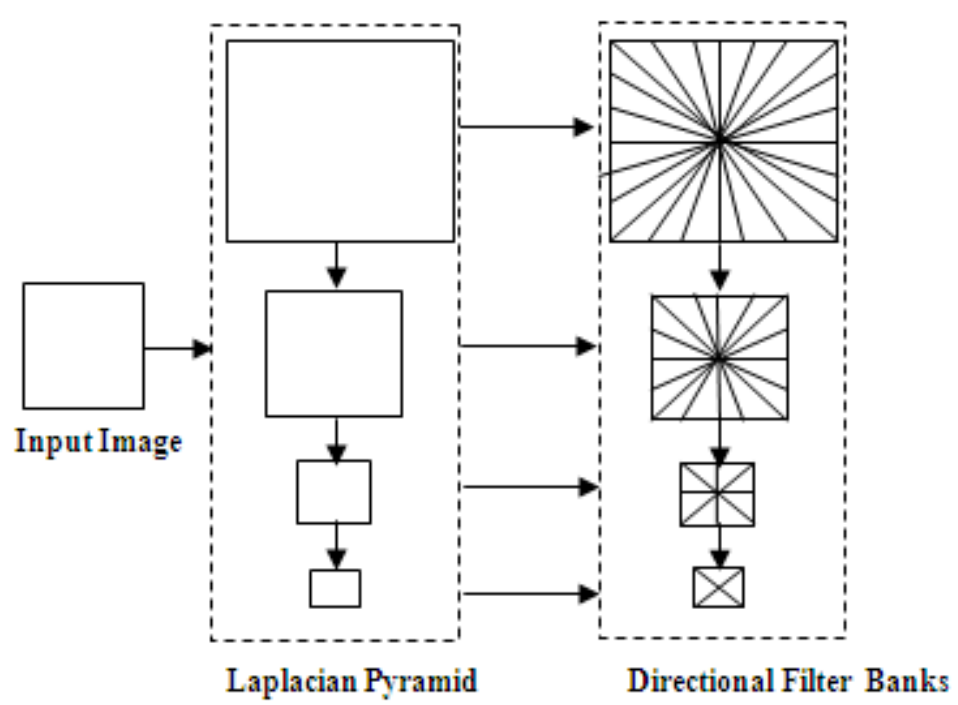

Fig. 1. The Contourlet Transform Framework

\section{The Proposed Method}

We know that edges and boundaries are salient features of images and required for better visual representation of images. Hence, we have proposed a new edge preserving image fusion method using contourlet transform. As contourlet transform has high degree of directionality and captures geometric structures in better way, it leads to a better detailed representation of images. The proposed method uses contourlet transform with edge preservation for fusion of different source images. In the proposed fusion method, first, the source images are decomposed using contourlet transform to obtain contourlet coefficients, followed by edge point computation using sobel gradient operator. Secondly, we preserve contourlet coefficients that correspond to edge points in the source images. Then we apply maximum fusion rule on remaining contourlet coefficients to obtain coefficients for fused image. Reconstruction of the obtained contourlet coefficients will result in fused image.

The steps of the proposed fusion algorithm can be summarized as follows:

1. Decompose source images $I_{1}(x, y)$ and $I_{2}(x, y)$ using Contourlet Transform (CT) to obtain contourlet coefficients $C_{1}(x, y)$ and $C_{2}(x, y)$ respectively. 


$$
\begin{aligned}
C_{1}(x, y) & =C T\left[I_{1}(x, y)\right] \\
\text { and } C_{2}(x, y) & =C T\left[I_{2}(x, y)\right]
\end{aligned}
$$

2. Use sobel operator to compute edge points $E_{1}(x, y)$ and $E_{2}(x, y)$ of contourlet coefficients $C_{1}(x, y)$ and $C_{2}(x, y)$ for source images $I_{1}(x, y)$ and $I_{2}(x, y)$ respectively.

3. Preserve edge points in the fused coefficient set $C(x, y)$ as following:

$$
C(x, y)=\left\{\begin{array}{l}
C_{1}(x, y), \text { if } E_{1}(x, y)=1 \\
C_{2}(x, y), \text { if } E_{2}(x, y)=1
\end{array}\right.
$$

4. Apply maximum fusion rule on remaining contourlet coefficients to obtain coefficients for fused image i.e.

$$
C(x, y)=\left\{\begin{array}{l}
C_{1}(x, y), \text { if }\left|C_{1}(x, y)\right|>\left|C_{2}(x, y)\right| \\
C_{2}(x, y), \text { if }\left|C_{2}(x, y)\right|>\left|C_{1}(x, y)\right|
\end{array}\right.
$$

5. Reconstruction of $C(x, y)$ provides fused image $F(x, y)$.

$$
F(x, y)=\text { Inverse } C T[C(x, y)]
$$

\section{$4 \quad$ Results and Discussions}

This section gives visual and quantitative comparison of the proposed method with DWT and SWT based fusion techniques. We have performed the proposed fusion method over several image data sets and fusion results for representative multifocus and medical image datasets are shown in figure 2, figure 3 and figure 4 . The proposed edge preserving fusion method is compared with DWT [15, 16] and SWT [17] based fusion techniques in which fusion is performed using average, average-maximum and maximum fusion rules. The proposed method preserves contourlet coefficients that correspond to edge points in the source images. This edge preserving step in the proposed method provides better edge information in the fused image that can be viewed from the visual representation of results shown in the figure 2, figure 3 and figure 4.

For objective evaluation of the proposed fusion method, we have used well known fusion measures [24, 25] - entropy, standard deviation, mutual information (MI) and $Q_{A B}^{F}$. Higher values of these fusion metrics will imply better fused image. To compare the proposed fusion method with DWT and SWT based fusion methods, we have computed above mentioned fusion measures and tabulated them in table 1, table 2 and table 3 for multifocus and medical image data sets. By observing table 1, one can easily found that fused image obtained by the proposed fusion method has higher values for three fusion metrics (entropy, mutual information and $Q_{A B}^{F}$ ) and very closed (62.1098) to maximum (62.4296) for standard deviation metric. By observing table 2, again we have highest values for entropy (7.1266) and mutual information (0.5643). Similarly in table 3 , the proposed method has the highest values for three metrics 
(entropy, mutual information and $Q_{A B}^{F}$ ) and closer to standard deviation metric for maximum fusion case. These comparisons show that the proposed edge preserving fusion method has better visual representation than DWT and SWT based fusion techniques as well as has higher values for different fusion metrics. Therefore, it can be concluded that the proposed edge preserving fusion using Contourlet Transform is able to preserve detail features of source images into resulting fused image and performs better than DWT and SWT based fusion methods.

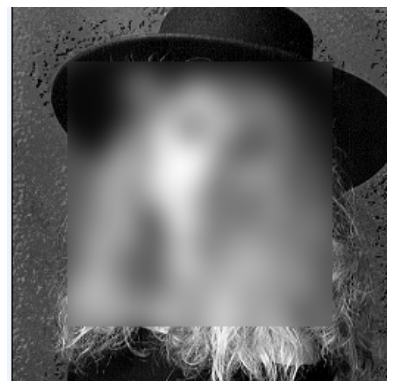

(a)

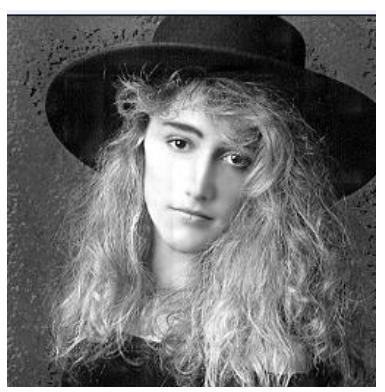

(d)

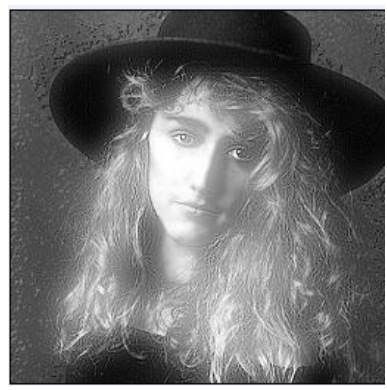

(g)

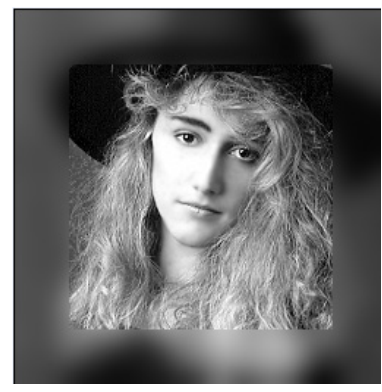

(b)

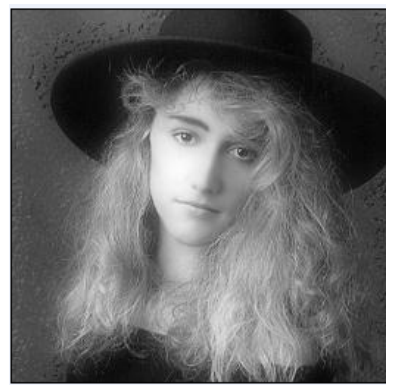

(e)

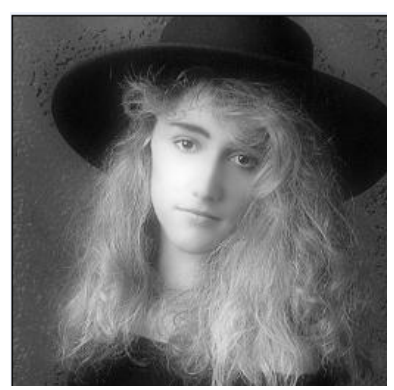

(h)

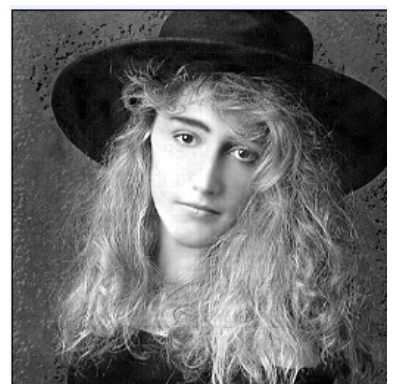

(c)

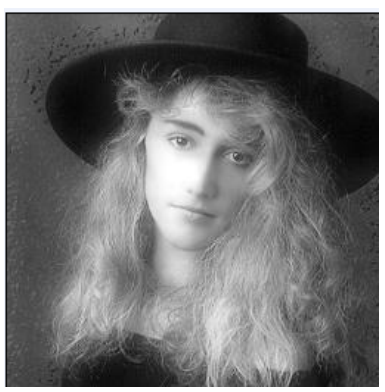

(f)

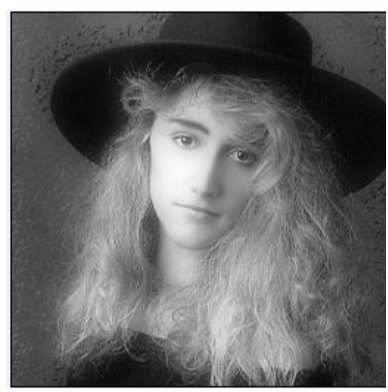

(i)

Fig. 2. Fusion results for multifocus images. (a). Source image 1, (b). Source image 2, (c). Fused image by the proposed fusion method, (d). DWT maximum fused image, (e). DWT average fused image, (f). DWT average-maximum fused image, (g). SWT maximum fused image, (h). SWT average fused image and (i). SWT average-maximum fused image. 


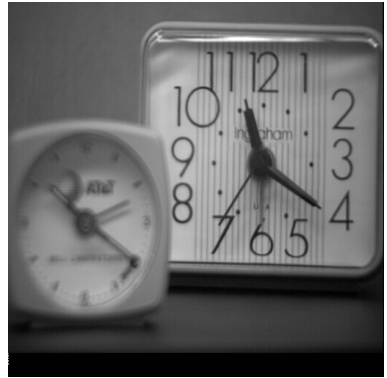

(a)

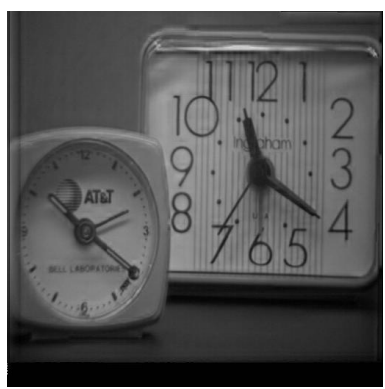

(d)

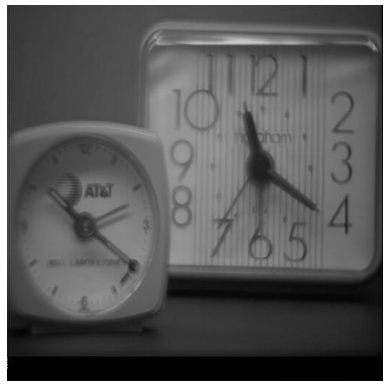

(g)

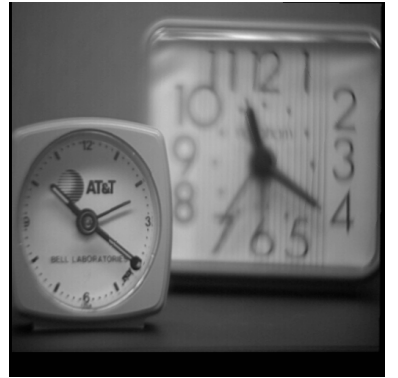

(b)

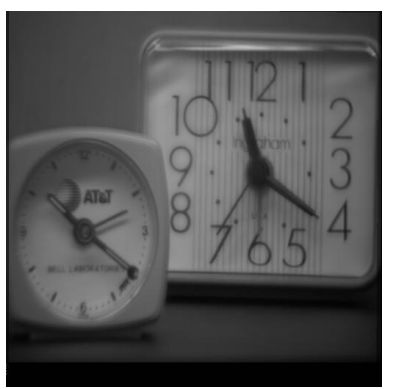

(e)

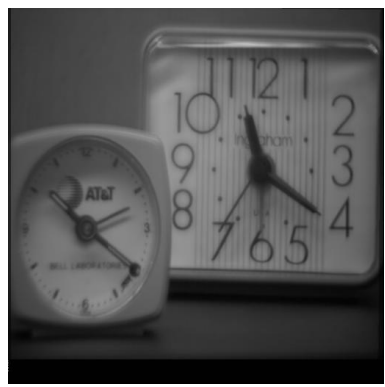

(h)

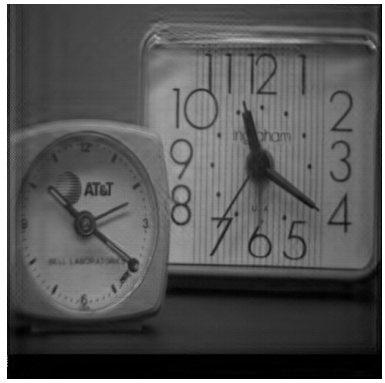

(c)

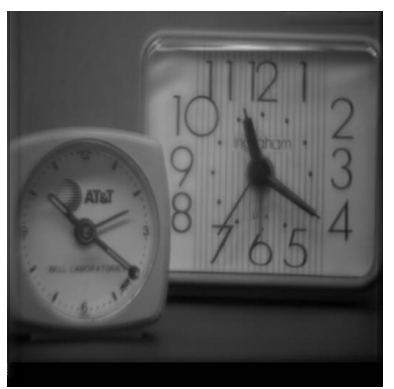

(f)

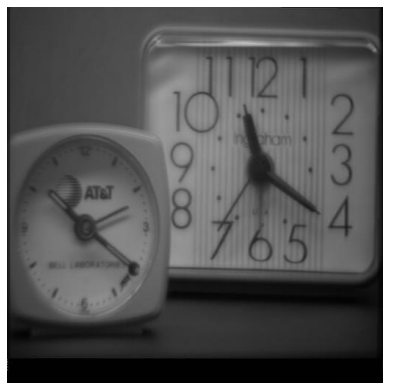

(i)

Fig. 3. Fusion results for Clock images. (a). Source image 1, (b). Source image 2, (c). Fused image by the proposed fusion method, (d). DWT maximum fused image, (e). DWT average fused image, (f). DWT average-maximum fused image, (g). SWT maximum fused image, (h). SWT average fused image and (i). SWT average-maximum fused image. 


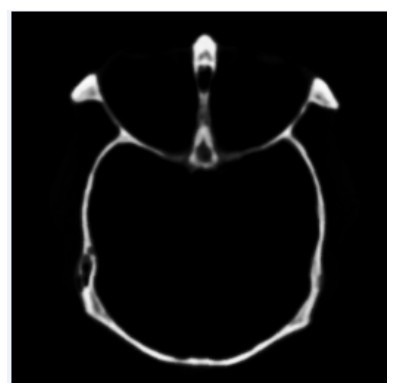

(a)

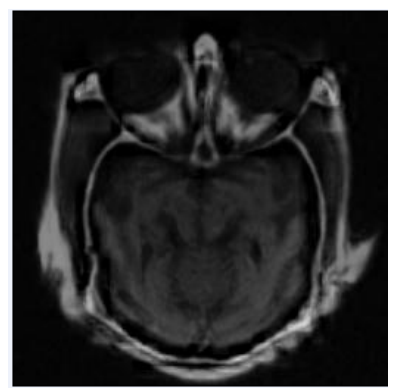

(d)

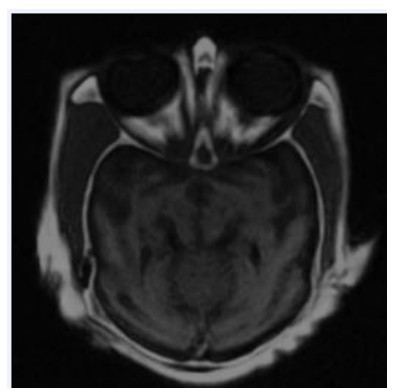

(g)

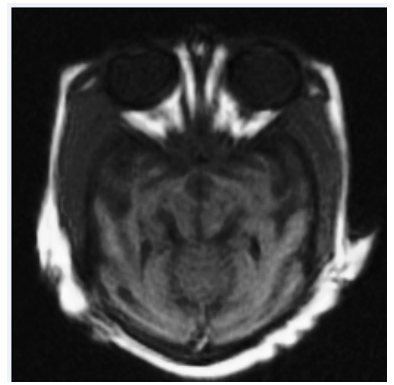

(b)

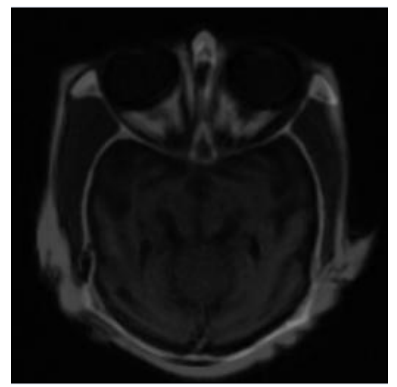

(e)

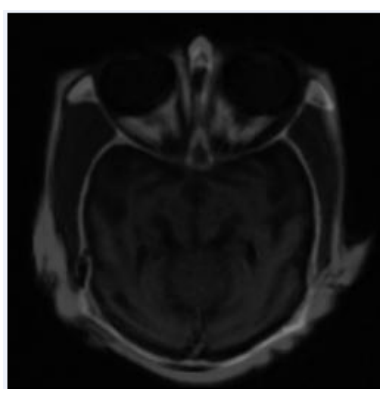

(h)

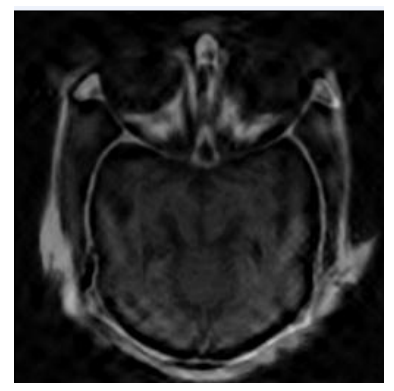

(c)

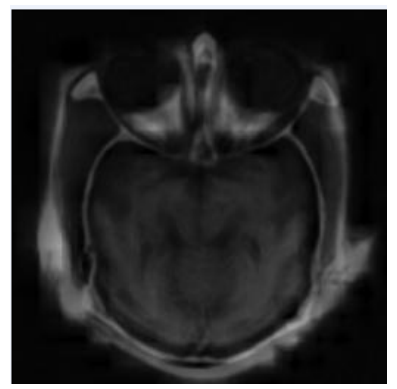

(f)

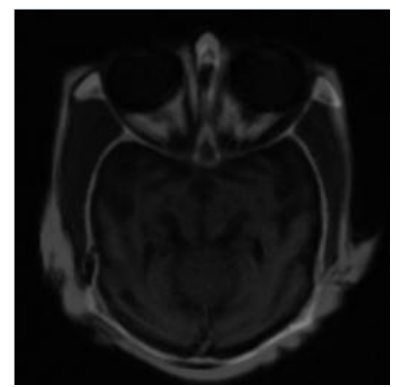

(i)

Fig. 4. Fusion results for medical images. (a). Source image 1, (b). Source image 2, (c). Fused image by the proposed fusion method, (d). DWT maximum fused image, (e). DWT average fused image, (f). DWT average-maximum fused image, (g). SWT maximum fused image, (h). SWT average fused image and (i). SWT average-maximum fused image. 
Table 1. Fusion performance evaluation for Multifocus Image Data Set

\begin{tabular}{|c|c|c|c|c|}
\hline Fusion Method & Entropy & $\begin{array}{c}\text { Standard } \\
\text { Deviation }\end{array}$ & MI & $Q_{A B}^{F}$ \\
\hline CT(EDGE) & $\mathbf{7 . 7 7 0 7}$ & 62.1098 & $\mathbf{4 . 5 2 2 2}$ & $\mathbf{0 . 6 6 9 7}$ \\
\hline DWT(MAX) & 7.7688 & $\mathbf{6 2 . 4 2 9 6}$ & 4.2728 & 0.6307 \\
\hline DWT(AVG) & 7.6059 & 56.4075 & 4.5725 & 0.3330 \\
\hline DWT(AVGMAX) & 7.6709 & 57.9649 & 4.5424 & 0.3385 \\
\hline SWT(MAX) & 7.7701 & 60.9528 & 4.4907 & 0.4744 \\
\hline SWT(AVG) & 7.6090 & 56.4817 & 4.5786 & 0.3302 \\
\hline SWT(AVGMAX) & 7.6873 & 58.0404 & 4.3518 & 0.5000 \\
\hline
\end{tabular}

Table 2. Fusion performance evaluation for Clock Image Data Set

\begin{tabular}{|c|c|c|c|c|}
\hline Fusion Method & Entropy & $\begin{array}{c}\text { Standard } \\
\text { Deviation }\end{array}$ & MI & $Q_{A B}^{F}$ \\
\hline CT(EDGE) & $\mathbf{7 . 1 2 6 6}$ & 40.5562 & 5.5334 & $\mathbf{0 . 5 6 4 3}$ \\
\hline DWT(MAX) & 7.0328 & $\mathbf{4 0 . 6 8 5 4}$ & 5.8496 & 0.5612 \\
\hline DWT(AVG) & 6.9687 & 39.6026 & 6.5894 & 0.4980 \\
\hline DWT(AVGMAX) & 6.9794 & 39.5327 & 6.2704 & 0.4979 \\
\hline SWT(MAX) & 6.9967 & 40.3693 & $\mathbf{6 . 9 9 7 1}$ & 0.4360 \\
\hline SWT(AVG) & 6.9689 & 39.6728 & 6.5973 & 0.4946 \\
\hline SWT(AVGMAX) & 6.9904 & 39.6661 & 6.5528 & 0.4899 \\
\hline
\end{tabular}

Table 3. Fusion performance evaluation for Medical Image Data Set

\begin{tabular}{|c|c|c|c|c|}
\hline Fusion Method & Entropy & $\begin{array}{c}\text { Standard } \\
\text { Deviation }\end{array}$ & MI & $Q_{A B}^{F}$ \\
\hline CT(EDGE) & $\mathbf{6 . 3 3 2 6}$ & 32.0629 & $\mathbf{3 . 6 4 2 0}$ & $\mathbf{0 . 7 7 8 7}$ \\
\hline DWT(MAX) & 6.1270 & 27.3918 & 2.0188 & 0.3617 \\
\hline DWT(AVG) & 5.1009 & 18.6230 & 2.9370 & 0.3325 \\
\hline DWT(AVGMAX) & 6.1270 & 27.3918 & 2.0188 & 0.3617 \\
\hline SWT(MAX) & 5.9902 & $\mathbf{3 2 . 9 0 1 4}$ & 3.5055 & 0.6805 \\
\hline SWT(AVG) & 5.1164 & 18.6744 & 2.9766 & 0.3292 \\
\hline SWT(AVGMAX) & 5.1912 & 18.8342 & 2.9618 & 0.3737 \\
\hline
\end{tabular}

\section{Conclusions}

In the present work, we have proposed a new edge preserving fusion method using Contourlet Transform. As Contourlet Transform has higher directionality and it captures smooth contours. Contourlet Transform based fusion using edge preservation is capable to preserve edge values in more efficient way. Experiments are performed over three set of image data sets (multifocus and medical). Visual representation of 
experimental results indicate that the proposed fusion method is better than wavelet transform (DWT and SWT) based fusion methods and provides better visual representation of fused image. Also we have verified the goodness of the proposed fusion method with well known fusion measures (entropy, standard deviation, mutual information $(\mathrm{MI})$ and $Q_{A B}^{F}$ ). This comparison also proved that the proposed edge preserving fusion method using Contourlet Transform is better than wavelet transform (DWT and SWT) based fusion methods.

Acknowledgements. This work was supported in part by the Department of Science and Technology, New Delhi, India, under grant no. SR/FTP/ETA-023/2009 and the University Grants Commission, New Delhi, India, under grant no. 36- 246/2008(SR).

\section{References}

1. Goshtasby, A., Nikolov, S.G.: Image Fusion: Advances in the state of the art, Guest editorial. Information Fusion 8(2), 114-118 (2007)

2. Toet, A., Hogervorst, M.A., Nikolov, S.G., Lewis, J.J., Dixon, T.D., Bull, D.R., Canagarajah, C.N.: Towards cognitive image fusion. Information Fusion 11(2), 95-113 (2010)

3. Pajares, G., Cruz, J.M.: A wavelet-based image fusion tutorial. Pattern Recognition 37(9), 1855-1872 (2004)

4. Darasthy, B.V.: Information fusion in the realm of medical applications - A bibliographic glimpse at its growing appeal. Information Fusion 13(1), 1-9 (2012)

5. Simone, G., Farina, A., Morabito, F.C., Serpico, S.B., Bruzzone, L.: Image fusion techniques for remote sensing applications. Information Fusion 3(1), 3-15 (2002)

6. Ranchin, T., Aiazzi, B., Alparone, L., Baronti, S., Wald, L.: Image fusion- the ARSIS concepts and some successful implementation schemes. ISPRS Journal of Photogrammetry \& Remote Sensing 58(1-2), 4-18 (2003)

7. Janczak, D., Sankowski, M.: Data fusion for ballistic targets tracking using least squares. AEU- International Journal of Electronics and Communications (2011) (article in press), http://dx.doi.org/10.1016/j .aeue.2011.11.003

8. Xue, Z., Blum, R.S., Li, Y.: Fusion of Visual and IR Images for Concealed Weapon Detection. In: Proceedings of International Conference on Image Fusion (ISIF), vol. 2, pp. 1198-1205 (2002)

9. Ross, A., Jain, A.: Information Fusion in Biometrics. Pattern Recognition Letters 24(13), 2115-2125 (2003)

10. Pohl, C., Genderen, J.L.V.: Multisensor image fusion in remote sensing: concept, methods and applications. International Journal of Remote Sensing 19(5), 823-854 (1998)

11. Li, H., Manjunath, B.S., Mitra, S.K.: Multisensor image fusion using the wavelet transform. Graphical Models and Image Processing 57(3), 235-245 (1995)

12. Amolins, K., Zhang, Y., Dare, P.: Wavelet based image fusion techniques—an introduction, review and comparison. ISPRS Journal of Photogrammetry \& Remote Sensing 62(4), 249-263 (2007)

13. Singh, R., Srivastava, R., Prakash, O., Khare, A.: DTCWT based Multimodal Medical Image Fusion. In: Proceedings of International Conference on Signal, Image and Video Processing (ICSIVP 2012), IIT Patna, Patna, pp. 403-407 (2012) 
14. Singh, R., Srivastava, R., Prakash, O., Khare, A.: Mixed scheme based multimodal medical image fusion using Daubechies Complex Wavelet Transform. Accepted to appear in Proceedings of International Conference on Informatics, Electronics \& Vision (IEEE/IAPR ICIEV 2012), Dhaka, Bangladesh, May 18-19 (2012)

15. Shangli, C., Junmin, H.E., Zhongwei, L.: Medical Images of PET/CT Weighted Fusion Based on Wavelet Transform. Bioinformatics and Biomedical Engineering, 2523-2525 (2008)

16. Singh, R., Khare, A.: A Wavelet Based Multimodal Medical Image Fusion. In: Proceedings of International Symposium on Medical Imaging: Perspectives on Perception and Diagnostics, in Conjunction with Seventh Indian Conference on Computer Vision, Graphics and Image Processing (ICVGIP-2010), IIT- Delhi, New Delhi, December 9-10 (2010)

17. Singh, R., Vatsa, M., Noore, A.: Multimodal Medical Medical Image Fusion using Redundant Wavelet Transform. In: Proce. of Seventh International Conference on Advances in Pattern Recognition, pp. 232-235 (2009)

18. Do, M.N., Vetterli, M.: The Contourlet Transform: an efficient directional multiresolution image representation. IEEE Transactions on Image Processing 14(12), 2091-2106 (2005)

19. Do, M.N., Vetterli, M.: Contourlets: a directional multiresolution image representation. In: Proceedings of International Conference of Image Processing, pp. 357-360 (2002)

20. Do, M.N., Vetterli, M.: Contourlets. In: Stoeckler, J., Welland, G.V. (eds.) Beyond Wavelets, pp. 1-27. Academic Press, New York (2002)

21. Tang, L., Zhao, Z.: The Wavelet-based Contourlet Transform for Image Fusion. In: Proceedings of Eighth ACIS International Conference on Software Engineering, Artificial Intelligence, Networking and Parallel/Distributed Computing, pp. 59-64 (2007)

22. Asmare, M.H., Asirvadam, V.S., Iznita, L., Hani, A.F.M.: Image Enhancement by Fusion in Contourlet Transform. International Journal on Electrical Engineering and Informatics 2(1), 29-42 (2011)

23. Yang, L., Guo, B., Ni, W.: Multifocus Image Fusion Algorithm based on Contourlet Transform and Region Statistics. In: Fourth International Conference on Image and Graphics (IJIG), pp. 707-712 (2007)

24. Kotwal, K., Chaudhuri, S.: A novel approach to quantitative evaluation of hyperspectral image fusion techniques. Information Fusion (2011) (article in press),

http://dx.doi.org/10.1016/j.inffus.2011.03.008

25. Xydeas, S., Petrovic, V.: Objective Image Fusion Performance Measure. Electronics Letters 36(4), 308-309 (2000) 